\title{
Antiretroviral Therapy Adherence, Medication Use, and Health Care Costs During 3 Years of a Community Pharmacy Medication Therapy Management Program for Medi-Cal Beneficiaries with HIV/AIDS
}

\author{
Jan D. Hirsch, RPh, PhD; Marco Gonzales, PharmD; Ashley Rosenquist, PharmD; \\ Teresa Ann Miller, PharmD; Todd P. Gilmer, PhD; and Brookie M. Best, PharmD, MAS
}

\begin{abstract}
BACKGROUND: The types of pharmacist-provided medication therapy management (MTM) services provided to patients with human immunodeficiency virus/acquired immune deficiency syndrome (HIV/AIDS) and the effects of MTM on medication adherence and patient outcomes have only recently begun to be studied. Although available studies suggest that patients receiving MTM services have better antiretroviral therapy (ART) adherence and outcomes, only 1 study has examined a large group of patients with HIV/AIDS, and none has examined adherence or outcomes for more than 1 year. A pilot program conducted by the California Department of Health Care Services (DHCS) and Medi-Cal (California's Medicaid program) provided an opportunity to examine ART adherence and outcomes in a large patient population receiving MTM services in community pharmacies over 3 years.
\end{abstract}

OBJECTIVES: To examine an HIV/AIDS pharmacy MTM compensation pilot program over a 3-year period (2005-2007) in a sample of Medi-Cal beneficiaries by describing the associations between use of pilot pharmacies and (a) adherence to ART regimens; (b) medication utilization, including number and type of ART medication regimens and use of contraindicated ART regimens; (c) occurrence of opportunistic infections; and (d) all-cause pharmacy and medical costs.

METHODS: This was a cohort study examining Medi-Cal pharmacy and medical claims data (2005-2007) for patients with HIV/AIDS who were served by pilot pharmacies versus other (nonpilot) pharmacies. The study groups, pilot and nonpilot pharmacy patients with HIV/AIDS, consisted of Medi-Cal beneficiaries aged 18 years or older as of January 1, 2005, who were continuously enrolled from January 1, 2004, through December 31, 2007, and who received both a diagnosis of HIV/AIDS and at least 1 ART pharmacy claim during both the index period (2004) and the study period (January 1, 2005, through December 31, 2007). Pilot pharmacy patients were identified as having filled $50 \%$ or more of their ART prescriptions each year at 1 of the 10 pilot pharmacies. Patients for whom comprehensive medication data were not available, including those enrolled in managed care plans and/or Medicare, were excluded. Adherence was defined as a medication possession ratio (MPR) of $80 \%-120 \%$ and excess medication fills as MPR greater than $120 \%$. Logistic regression was used to investigate the factors associated with adherence. Comparisons were made between groups using bivariate statistics (Pearson chi-square for categorical variables and t-tests for continuous variables). For comparisons of costs, generalized linear models were used including predictor variables for age, gender, and race/ethnicity.
RESULTS: The study sample consisted of 2,234 patients meeting the study inclusion criteria. The proportion of study patients receiving the majority of their prescription medications (ART plus non-ART) at pilot pharmacies was $19.7 \%$ in 2005 and increased to $27.6 \%$ in 2006 and $28.1 \%$ in 2007 . The demographic profile of pilot pharmacy patients was similar to that of patients receiving medications at nonpilot pharmacies, except that pilot pharmacies had a higher proportion of Latino patients (e.g., $19.7 \%$ vs. $14.9 \%$ in 2007 , respectively, $P=0.006$ ). A greater percentage of pilot than nonpilot pharmacy patients were adherent to their ART medication regimens (e.g., 2007: $69.4 \%$ vs. $47.3 \%$, respectively, $P<0.001$ ). After controlling for age, gender, and ethnicity/race in logistic regression analysis, use of a pilot pharmacy (odds ratio $[0 \mathrm{R}]=2.74,95 \% \mathrm{Cl}=2.44-3.10$ ) was the most important factor associated with likelihood of adherence. Each year, pilot pharmacy patients were more likely than nonpilot pharmacy patients to remain on a single type of ART regimen (e.g., 2007: $71.7 \%$ vs. $49.1 \%$, respectively, $P<0.001$ ) and less likely to have excess fills (e.g., 2007: $12.9 \%$ vs. $35.5 \%$, respectively, $P<0.001$ ) and to use contraindicated regimens (e.g., $2007: 8.9 \%$ vs. $12.2 \%$, respectively, $P=0.027$ ). The percentages of patients experiencing opportunistic infections were similar between groups each year, approximately $35 \%(P=0.809-0.945)$. In the generalized linear model analyses, the between-group differences in predicted mean (standard error [SE]) total health care costs per patient were not significantly different in any year (e.g., 2007: \$38,983 [\$1,023] vs. $\$ 38,856$ [ $\$ 633$ ], respectively, $P=0.915)$. In each year, predicted nonART medication costs were approximately $30 \%-40 \%$ greater in the pilot pharmacy than nonpilot pharmacy group (e.g., 2007: $\$ 10,815$ [\$538] vs. $\$ 8,190$ [\$252], respectively, $P<0.001)$; however, predicted expenditures for inpatient services were significantly lower (e.g., 2007: \$3,083 [\$293] vs. $\$ 5,186$ [ $\$ 300$ ], respectively, $P<0.001$ ). Payment from the DHCS Medi-Cal program for MTM services was approximately $\$ 1,000$ per pilot pharmacy patient per year.

CONCLUSIONS: Over a 3-year period, patients at pilot pharmacies consistently had higher medication adherence rates, were more likely to remain on a single type of ART regimen throughout the year, had fewer excess fills, and used fewer contraindicated regimens than nonpilot pharmacy patients. There were no significant differences in mean total cost per patient per group, and the additional MTM services payment added less than $3 \%$ to the total cost.

\section{J Manag Care Pharm. 2011;17(3):213-23}

Copyright $\odot 2011$, Academy of Managed Care Pharmacy. All rights reserved. 


\section{Antiretroviral Therapy Adherence, Medication Use, and Health Care Costs During 3 Years of a Community Pharmacy Medication Therapy Management Program for Medi-Cal Beneficiaries with HIV/AIDS}

\section{What is already known about this subject}

- A significant correlation between improved antiretroviral therapy (ART) adherence and reduced viral load has been demonstrated for patients with human immunodeficiency virus/acquired immune deficiency syndrome (HIV/AIDS) in several studies. For example, a study in a Veterans Affairs Medical Center found that each adherence rate increase of $10 \%$ was associated with a viral load decrease of $0.12 \log _{10}\left(95 \% \mathrm{CI}=0.01 \log _{10}-0.23 \log _{10}\right)$ copies per milliliter $(\mathrm{mL})$.

- Two published studies suggest that HIV/AIDS patients receiving pharmacist-provided medication therapy management (MTM) services have better ART adherence and outcomes; however, these studies used small sample sizes and were conducted in specialized clinics, not community pharmacies.

- A study of the first year (2005) of a Medi-Cal pilot program that provided compensation for pharmacist-provided MTM services for Medi-Cal beneficiaries, including Medicare dual-eligibles, found that pilot community pharmacy patients were more likely to be adherent to ART medications ( $56.3 \%$ vs. $38.1 \%, P<0.001$ ); to use protease inhibitor-based ART medication regimens $(63.8 \%$ vs. $54.8 \%, P<0.001)$; and to remain on a single type of ART regimen throughout the study year $(56.8 \%$ vs. $34.2 \%, P<0.001)$ and were less likely to use contraindicated regimens (11.6\% vs. $16.6 \%$, $P<0.001)$ compared with nonpilot pharmacy patients.

\section{What this study adds}

- The present study examined the second and third year of the Medi-Cal MTM services pilot program excluding Medicare dualeligibles, finding that the results observed in the first evaluation year (2005) were sustained in the group of Medi-Cal patients who continued to receive care at pilot pharmacies from 2005 through 2007.

- Compared with nonpilot pharmacy patients, pilot pharmacy patients were (a) more adherent to their ART medication regimens, (b) more likely to remain on a single type of ART regimen, (c) less likely to have excess fills, and (d) less likely to use contraindicated regimens; however, there was no difference in the proportion of patients experiencing opportunistic infections (about 35\% in both groups).

- Non-ART medication costs were approximately 30\%-40\% greater in the pilot pharmacy group each year, and the difference was primarily and consistently attributable to greater use of gastrointestinal agents, analgesics, and psychotherapeutic medications; however, expenditures for inpatient services were significantly lower for pilot pharmacy than nonpilot pharmacy patients each year (e.g., 2007: mean [standard error, SE] \$3,083 [\$293] vs. $\$ 5,186$ [\$300], respectively, $P<0.001$ ).

- The total mean [SE] annual cost per patient was not significantly different for pilot versus other pharmacy patients in any year (e.g., 2007: \$38,983 [\$1,023] vs. \$38,856 [\$633], respectively, $P=0.915$ ); and the additional MTM services payment of approximately $\$ 1,000$ per patient added less than 3\% to the total cost per patient per year
A ntiretroviral therapy (ART) regimens for patients with human immunodeficiency virus/acquired immune deficiency syndrome (HIV/AIDS) are complex. ART regimens often contain multiple drugs with various dosing schedules, different storage requirements, and undesirable side effect profiles. ${ }^{1}$ ART regimens comprise multiple medications from at least 2 of the 3 main drug classes including protease inhibitors (PI), non-nucleoside reverse transcriptase inhibitors (NNRTI), and nucleoside or nucleotide reverse transcriptase inhibitors (NRTI). ${ }^{1}$ However, available anti-HIV drugs are limited. Once a regimen fails and those specific drugs can no longer be used (i.e., are inactive against the virus), the choices available for a new 2-drug regimen become limited. Thus, remaining on a particular medication regimen that successfully suppresses viral replication is important because it slows disease progression and preserves options for future treatment. Although boosted PI-based therapy (i.e., low-dose ritonavir given concomitantly to boost the exposure to the primary PI) is the preferred regimen for both treatment-naïve and treatment-experienced patients, this regimen also has the least tolerable side effects. ${ }^{1}$ Some regimens are contraindicated due to their excess toxicity or inferior efficacy compared with recommended regimens. ${ }^{1}$

Achieving optimal therapeutic outcomes, such as reduced viral load, reduction of drug resistance, and improved survival, requires strict adherence to ART regimens. ${ }^{2-4} \mathrm{~A}$ significant correlation between better medication adherence and reduced viral load has been demonstrated in several studies using pharmacy claims data. ${ }^{5-7}$ For example, a study using pharmacy dispensing records in a free HIV clinic found that when adherence levels were below 95\%, the percentage of patients with viral loads consistently below 400 copies per milliliter (mL) fell below 60\%. ${ }^{6}$ A study in a Veterans Affairs Medical Center found that each adherence rate increase of $10 \%$ was associated with a viral load decrease of $0.12 \log _{10}$ (95\% confidence interval $\left.[\mathrm{CI}]=0.01 \log _{10}-0.23 \log _{10}\right)$ copies per $\mathrm{mL}^{7}$

The types of pharmacist-provided medication therapy management (MTM) services provided to patients with HIV/AIDS and the effects of MTM on medication adherence and patient outcomes have only recently begun to be studied. Two small studies conducted in HIV outpatient clinics reported positive outcomes associated with patient attendance at pharmacist-led clinics. ${ }^{8,9}$ The first reported that patients attending a pharmacist-led medication adherence clinic and at least 1 educational session from the clinical pharmacist $(n=80)$ experienced a significantly greater reduction in viral load at 6 and 12 months $(P<0.05)$ and improved medication adherence (refilling prescriptions on average every 31 vs. 50 days, $P<0.05$ ) compared with those not attending. ${ }^{8}$ The second study, without a comparison group, reported that patients attending a pharmacist-managed drug optimization clinic $(n=34)$ experienced 


\section{Antiretroviral Therapy Adherence, Medication Use, and Health Care Costs During 3 Years of a Community Pharmacy Medication Therapy Management Program for Medi-Cal Beneficiaries with HIV/AIDS}

significant improvement from baseline in CD4 + T-lymphocyte counts $(P<0.001)$ and viral loads $(P=0.004)$, and a significant decrease in the severity of ART-related toxicity $(P<0.001)$ over the study period (mean [standard deviation, SD] $=4$ [2] months). ${ }^{9}$

The largest published study of MTM services for patients with HIV/AIDS suggested improved patient adherence and medication usage patterns for patients using HIV/AIDS intensive community pharmacies participating in a special California Department of Health Care Services (DHCS) Medi-Cal (California's Medicaid program) pilot program that provided compensation to community pharmacists for MTM services.$^{10}$ Pharmacists practicing in participating pharmacies had continuing and/or advanced training in HIV/AIDS patient care and offered a range of MTM services. ${ }^{11}$ The most common MTM services were evaluation of patients' ability to adhere to medications, identifying and managing adverse drug reactions, tailoring ART regimens to meet special lifestyle needs, counseling when medication underuse or overuse was detected, refill reminder services, and referral to other medical services as needed. Results of the first year of the pilot program (2005) indicated that more pilot pharmacy patients were classified as adherent with their medication regimens compared with patients receiving care at other (nonpilot) pharmacies. ${ }^{10}$ In addition, fewer pilot pharmacy patients used contraindicated regimens, and pilot pharmacy patients were more likely to use PI-based ART medication regimens and to remain on a single type of ART therapy over the 1-year period. However, medical care utilization that may reflect differences in clinical outcomes (i.e., outpatient and hospitalization costs) and the rate of opportunistic infections were not significantly different between groups during the 1-year study period.

Although these studies suggest that HIV/AIDS patients receiving pharmacist-provided MTM services have better medication usage patterns (e.g., ART adherence) and clinical markers (e.g., viral load), only 1 study has examined a large group of HIV/AIDS patients, and none has examined adherence or outcomes for more than a single year. Follow-up for more than 1 year is important to determine if improved shortterm behaviors and medication therapy result in fewer adverse events and better clinical outcomes that may lead to reduced medical resource utilization. Evaluation of data from the second and third year of the Medi-Cal MTM services pilot program provided an opportunity to examine ART adherence and outcomes in a patient population using MTM services provided in community pharmacies over multiple years.

\section{Study Objectives}

The primary purpose of this study was to examine the association between use of pharmacies participating in a California DHCS Medi-Cal MTM pilot program and adherence to ART regimens in a sample of Medi-Cal beneficiaries with HIV/AIDS during a 3-year period (2005-2007). In addition, the study used pharmacy and medical claims data for patients of pilot and nonpilot pharmacies to describe and compare (a) medication utilization, including number and type of ART medication regimens and use of contraindicated ART regimens; (b) occurrence of opportunistic infections; and (c) all-cause pharmacy and medical costs in each year.

\section{Methods}

\section{Description of the Intervention}

The intervention in this study was participation in the pilot Medi-Cal compensation program for MTM services for patients with HIV/AIDS. Participating pilot pharmacies were HIV/ AIDS-intensive community pharmacies offering a wide range of MTM services of their own choosing (i.e., not determined by the Medi-Cal pilot program). The program has been described elsewhere in detail, including results from the first year of the program. ${ }^{10,11}$ MTM services were self-reported by pharmacists, with each pilot pharmacy offering services beyond standard counseling, such as "evaluation of patients' ability to adhere to medications, in consultation with doctors and case managers;" "identifying and managing adverse drug reactions;" and "tailoring drug regimens to fit patient lifestyle or special needs." 11 In addition, the majority of pilot pharmacies also offered "individual appointments with pharmacists to discuss medication therapy;" "adherence packaging beyond any provided by manufacturer" (e.g., personalized blister packs for all ART medications); "identification of peer advocate to assist in medication adherence;" and "weekly telephone call or home visit after initiation of therapy." 11 Although many types of MTM services were offered, the proportion of patients utilizing each service varied. For example, "adherence packaging beyond any provided by the manufacturer" was offered by 4 of 7 pilot pharmacies responding to a survey by the authors of the present study, but the proportion of patients utilizing the service was estimated to be between $1 \%$ and $50 \% .{ }^{11}$

\section{Outcome Measures and Hypotheses}

We hypothesized that use of pilot pharmacies would be a significant predictor of greater ART adherence during the 3-year study period. To measure adherence for each year, MPR was calculated as the sum of days supply of ART divided by 365.26 days (Table 1). Adherence was defined as a medication possession ratio (MPR) of 80\%-120\%. Three additional MPR categories were defined: nonadherent (MPR less than 50\%), partially adherent (MPR 50\%-79\%), and excess fills (MPR more than $120 \%)$. A grouping for partially adherent was included due to its clinical significance; partial adherence can cause drug resistance, thus limiting the availability of future effective drug regimens. In addition, compared with patients using nonpilot pharmacies, we expected patients using pilot pharmacies to have (a) more rational ART medication strategies (i.e., greater 


\section{Antiretroviral Therapy Adherence, Medication Use, and Health Care Costs During 3 Years of a Community Pharmacy Medication Therapy Management Program for Medi-Cal Beneficiaries with HIV/AIDS}

\section{TABLE 1 Outcome Measures (2005-2007)}

\begin{tabular}{|c|c|}
\hline Variable & Description \\
\hline Number of pharmacy visits & Number of days on which the patient visited the pharmacy to fill ART prescription or prescriptions \\
\hline \multirow[t]{3}{*}{ ART adherence level (MPR) } & $\frac{\text { MPR }=\sum \text { number days supply ART for year }}{365.25 \text { days }}$ \\
\hline & $\begin{array}{l}\text { Nonadherent: MPR less than } 50 \% \\
\text { Partially adherent: MPR } 50 \%-79 \% \\
\text { Adherent: MPR } 80 \%-120 \% \\
\text { Excess fills: MPR more than } 120 \%\end{array}$ \\
\hline & $\begin{array}{l}\text { Calculated using medication with highest days supply on day when multiple prescriptions were filled to avoid double } \\
\text { counting of days }\end{array}$ \\
\hline $\begin{array}{l}\text { ART medication regimen } \\
\text { strategy }\end{array}$ & $\begin{array}{l}\text { Categories are mutually exclusive and are assigned the therapy with the greatest number of days supplied during the year } \\
\text { - Only } 1 \text { NRTI } \\
\text { - Multiple NRTI } \\
\text { - NRTI+NNRTI } \\
\text { - NRTI+PI, with or without NNRTI }\end{array}$ \\
\hline $\begin{array}{l}\text { Contraindicated ART } \\
\text { regimena }\end{array}$ & $\begin{array}{l}\text { - amprenavir+fosamprenavir } \\
\text { - atazanavir+indinavir } \\
\text { - zalcitabine in regimen } \\
\text { - emtricitabine+lamivudine } \\
\text { - stavudine + zidovudine } \\
\text { - didanosine + stavudine } \\
\text { - oquinavir alone } \\
\text { - only a single-class NRTI regimen } \\
\text { - only triple therapy NRTI-except if abacavir+zidovudine+lamivudine or tenofovir + zidovudine+lamivudine }\end{array}$ \\
\hline $\begin{array}{l}\text { Number of ART medication } \\
\text { regimen strategies }\end{array}$ & $\begin{array}{l}\text { Number ART regimen strategies }=\sum \text { regimen strategies } \\
\text { - Regimen strategies: each prescription fill categorized as a single ART, multiple ART, NNRTI, or PI regimen strategy } \\
\text { - Regimen strategies counted only once: (e.g., if patient switched from single ART to NNRTI and back to ART, number of regi- } \\
\text { men strategies=2) }\end{array}$ \\
\hline Opportunistic infection ${ }^{10}$ & See list in previously published study \\
\hline Total medication cost & Paid claims amount for all prescription medications \\
\hline ART medication cost & Paid claims amount for ART medications (single agent or in combination) \\
\hline Non-ART medication cost & Total medication cost minus ART medication cost \\
\hline Medical costs ${ }^{b}$ & $\begin{array}{l}\text { Paid claims amounts for: } \\
\text { Inpatient } \\
\text { Hospital outpatient (includes emergency department) } \\
\text { Outpatient } \\
\text { Mental health } \\
\text { Lab/x-ray } \\
\text { AIDS Waiver Programc }\end{array}$ \\
\hline
\end{tabular}

aU.S. Department of Health and Human Services, Panel on Antiretroviral Guidelines for Adults and Adolescents, Table 8, page 65. ${ }^{1}$ Note: didanosine + tenofovir, which is on the current list of contraindicated regimens, was not included in the present study because it was not listed in the 2006 guidelines relevant to the study period.

${ }^{b}$ Medical costs were assigned to service categories using codes indicating type of service (i.e., Medi-Cal vendor codes).

cUnder contract with the Department of Health Services agencies to provide home and community-based services as an alternative to nursing facility care or hospitalization.

AIDS = acquired immune deficiency syndrome; ART = antiretroviral therapy; $M P R=$ medication possession ratio; NNRTI=non-nucleoside reverse transcriptase inhibitor; NRTI = nucleoside or nucleotide reverse transcriptase inhibitor; $P I=$ protease inhibitor

use of PI-based ART regimens, fewer medication changes, fewer contraindicated regimens); (b) fewer opportunistic infections in each study year; and (c) lower total costs by study year 3. Specific outcome measures are presented in Table 1.

\section{Study Design and Patient Selection}

This was a cohort study examining Medi-Cal pharmacy and medical claims data for patients with HIV/AIDS served by pilot versus nonpilot pharmacies in the state of California during the calendar years 2005-2007. The University of California San
Diego and the California Health and Human Services Agency Committee for the Protection of Human Subjects approved all study procedures.

The study sample consisted of Medi-Cal beneficiaries aged 18 years or older as of January 1, 2005, who were continuously enrolled from January 1, 2004, through December 31, 2007, and diagnosed with HIV/AIDS, identified by receipt of at least 1 prescription for ART and at least 1 medical claim with a primary or secondary HIV/AIDS-related diagnosis (International Classification of Diseases, Ninth Revision, Clinical Modification 


\section{Antiretroviral Therapy Adherence, Medication Use, and Health Care Costs During 3 Years of a Community Pharmacy Medication Therapy Management Program for Medi-Cal Beneficiaries with HIV/AIDS}

\section{FIGURE 1 Patient Selection from Medi-Cal Claims Database}

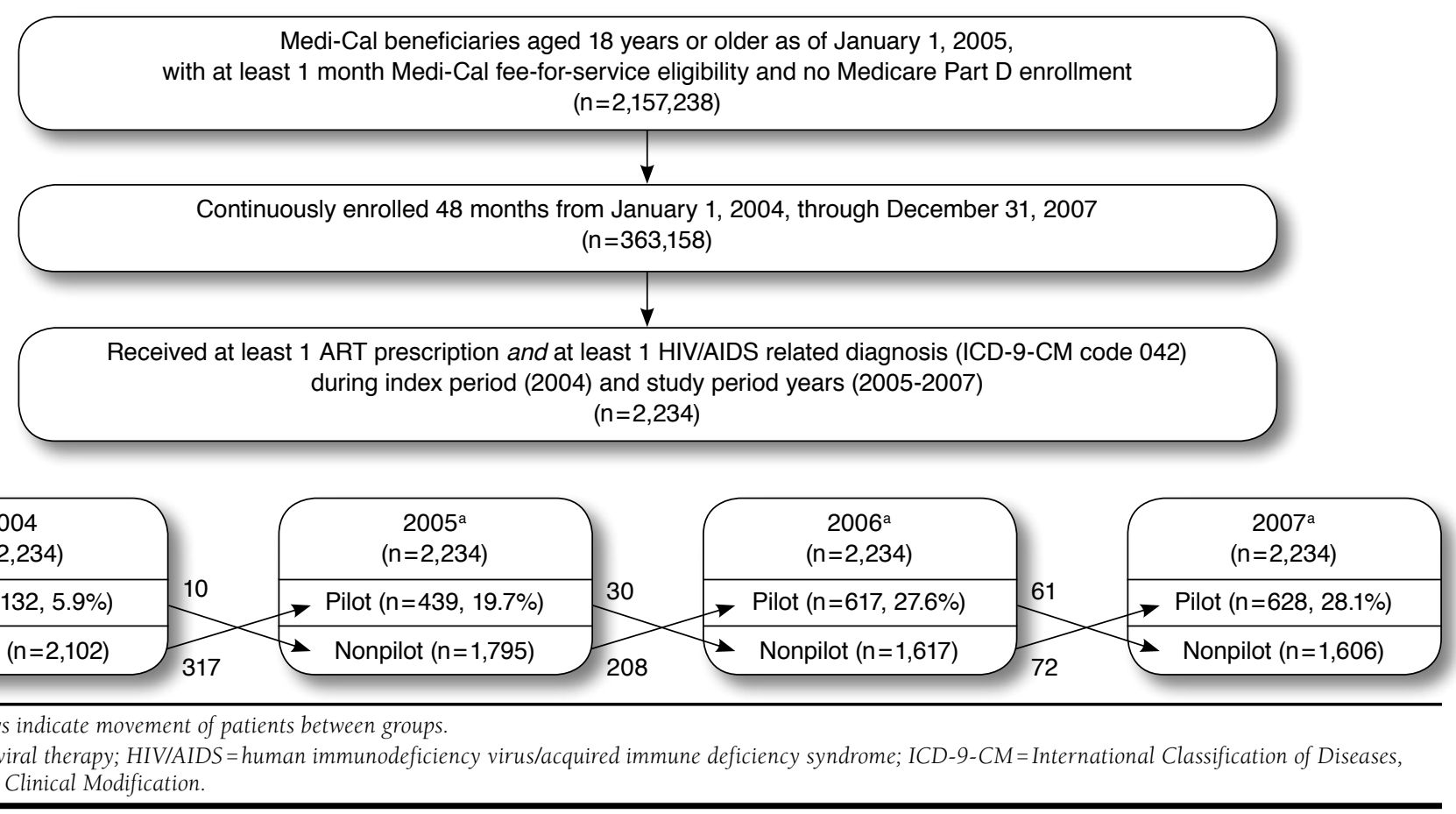

code $[\mathrm{ICD}-9-\mathrm{CM}]=042.0$ ) during both the index period (from January through December 2004) and the study period years (from January 2005 through December 2007). The ART medications included in this cohort included all those that were approved by the U.S. Food and Drug Administration at any time during the study period (Appendix). Two medications (maraviroc and raltegravir) that were approved in the last half of 2007 were not included because the opportunity to utilize these agents was minimal. In each study year, patients were identified as pilot pharmacy patients if they filled $50 \%$ or more of their ART prescriptions at 1 of the 10 pilot pharmacies. Comparison group patients met the same inclusion and exclusion criteria as study patients except that they filled less than $50 \%$ of their ART prescriptions at 1 of the 10 pilot pharmacies. Individuals enrolled in managed care plans were excluded because comprehensive information on paid claims was not available for Medi-Cal participants enrolled in managed care. Notably, dual-eligible Medicare patients were excluded because they had been automatically switched to Medicare Part D for the majority of their prescription drugs as of January 1, 2006, and as a result, their medication claims data were not available for follow-up in the Medi-Cal database. Patients who died at any time from 2004 through 2007 did not meet the continuous eligibility requirement for sample inclusion and were therefore not represented in the sample.

\section{Data Analysis}

Descriptive statistics were calculated for all variables. Categorical variables were described with frequency distributions and continuous variables by means, medians, SDs, and ranges. Differences in categorical variables (gender, race/ethnicity, ART adherence, and ART medication category) between groups (pilot vs. other pharmacy patients) were assessed using the Pearson chi-square test. Differences in continuous variables (age and number of pharmacy visits to fill ART prescriptions) were compared between groups using t-tests. Binomial logistic regression with the patient-year as the unit of analysis (i.e., each patient contributed 3 observations) was used to investigate the factors associated with adherence (MPR of 80\%$120 \%$ ) versus any other MPR (i.e., combining patients who were nonadherent, partially adherent, or had excess fills into a single category). Independent variables included pilot pharmacy usage (yes vs. no), patient age, gender, and racelethnicity. Differences in costs were analyzed using generalized linear models assuming a gamma distribution and log link function; predictor variables for the models included age, gender, and race/ethnicity. Standardized estimates of mean annual costs were calculated for pilot and nonpilot pharmacies. Costs for the $\$ 9.50$ payment per prescription dispensed (for any medication, not just ART) paid to pilot pharmacies by DHCS were calculated separately. Statistical significance was set at $P<0.05$. All statistical analyses were performed using SAS version 9.13 (SAS Institute, Inc., Cary, NC). 


\section{Antiretroviral Therapy Adherence, Medication Use, and Health Care Costs During 3 Years of a Community Pharmacy Medication Therapy Management Program for Medi-Cal Beneficiaries with HIV/AIDS}

TABLE 2 HIV/AIDS Patient Demographics: Pilot and Nonpilot Pharmacies, 2005-2007

\begin{tabular}{|c|c|c|c|c|c|c|c|c|c|}
\hline & \multicolumn{3}{|c|}{2005} & \multicolumn{3}{|c|}{2006} & \multicolumn{3}{|c|}{2007} \\
\hline & Pilot & Nonpilot & $P$ Value a & Pilot & Nonpilot & $P$ Value a & Pilot & Nonpilot & $P$ Value ${ }^{a}$ \\
\hline Number of pharmacies & 10 & 5,359 & NA & 10 & 5,236 & NA & 10 & 5,328 & NA \\
\hline Number (\%) study patients & $439(19.6)$ & $1,795(80.3)$ & NA & $617(27.6)$ & $1,617(72.4)$ & NA & $628 \quad(28.1)$ & $1,606(71.9)$ & NA \\
\hline Mean [SD] (range) age in years & $\begin{array}{c}44.7 \quad[8.1] \\
(18-62)\end{array}$ & $\begin{array}{c}45.4 \quad[7.8] \\
(18-70)\end{array}$ & 0.096 & $\begin{array}{c}45.8 \quad[8.2] \\
(19-67)\end{array}$ & $\begin{array}{c}46.5 \quad[7.7] \\
(20-71)\end{array}$ & 0.074 & $\begin{array}{cc}47.0 & {[8.1]} \\
(20-64)\end{array}$ & $\begin{array}{c}47.4 \quad[7.8] \\
(21-72)\end{array}$ & 0.347 \\
\hline Number (\%) male & $295(67.2)$ & $1,293(72.0)$ & 0.204 & $405(65.6)$ & $1,153(71.3)$ & 0.011 & $419 \quad(66.7)$ & $1,140(71.0)$ & 0.056 \\
\hline \multicolumn{10}{|l|}{ Race/ethnicity number (\%) } \\
\hline Non-Latino White & $177(40.3)$ & $767(42.7)$ & 0.359 & $224(36.3)$ & $720(44.5)$ & 0.001 & $224 \quad(35.7)$ & $720(44.8)$ & 0.001 \\
\hline African American & $145(33.0)$ & $563(31.4)$ & 0.502 & $209(33.9)$ & 499 (30.9) & 0.171 & $217 \quad(34.6)$ & $491(30.6)$ & 0.069 \\
\hline Latino & $71(16.2)$ & $293(16.3)$ & 0.939 & $120(19.4)$ & $244(15.1)$ & 0.013 & $124 \quad(19.7)$ & $240(14.9)$ & 0.006 \\
\hline Other race/ethnicity & $46(10.5)$ & $172 \quad(9.6)$ & 0.571 & $64(10.4)$ & $154(9.5)$ & 0.546 & $63(10.0)$ & $155 \quad(9.7)$ & 0.785 \\
\hline
\end{tabular}

\section{Results}

The study sample consisted of 2,234 patients meeting the study inclusion criteria (Figure 1). The present study cohort (continuous eligibility for 4 years from January 1, 2004, through December 31, 2007), compared with the original cohort identified for the previously published 2005 analyses (continuous eligibility for 2004-2005), ${ }^{10}$ had a higher percentage of African Americans (33.0\% vs. $29.4 \%$ pilot pharmacies and $31.4 \%$ vs. $25.2 \%$ nonpilot pharmacies) and slightly lower percentages of non-Latino White patients ( $40.3 \%$ vs. $44.6 \%$ pilot pharmacies and $42.7 \%$ vs. $46.5 \%$ nonpilot pharmacies) and Latino patients (16.2\% vs. $17.6 \%$ pilot pharmacies and $16.3 \%$ vs. $19.9 \%$ nonpilot pharmacies). While still predominantly male, the percentage of males was lower in the present study compared with the previous study $(67.2 \%$ vs. $76.3 \%$ pilot pharmacies and $72.0 \%$ vs. $81.0 \%$ nonpilot pharmacies).

Most nonpilot pharmacy patients did not use pilot pharmacies to fill any ART prescriptions. For example, in 2007 1,428 (88.9\%) of nonpilot pharmacy patients filled no ART prescriptions at pilot pharmacies; 63 (3.9\%) used a pilot pharmacy for $25 \%-49 \%$ of their ART claims; and 115 (7.2\%) used a pilot pharmacy for $1 \%-24 \%$ of their ART claims. The proportion of cohort patients receiving the majority of their prescription medications (ART plus non-ART) at pilot pharmacies was $19.7 \%$ in 2005 and increased to $27.6 \%$ and $28.1 \%$ during 2006 and 2007 (Table 2). Pilot pharmacies had a slightly lower percentage of male patients than nonpilot pharmacies in 2006 and 2007 ( $P=0.011$ and $P=0.056$, respectively). The pilot pharmacy group also had a larger proportion of Latino patients compared with the nonpilot group in 2006 and 2007 (e.g., 2007: $19.7 \%$ vs. $14.9 \%$ respectively, $P=0.006)$ and a smaller percentage of non-Latino White patients (e.g., 2007: 35.7\% vs. $44.8 \%$ respectively, $P=0.001$ ).

The percentage of patients who were adherent with ART was significantly greater for pilot pharmacies than nonpilot pharmacies, with the difference being greater than 20 percentage points each year (e.g., 2007: 69.4\% vs. 47.3\% respectively, $P<0.001$; Table 3 ). A much smaller percentage of patients using pilot pharmacies had excess fills than patients using other pharmacies (e.g., 2007: 12.9\% vs. 35.5\% respectively, $P<0.001)$. In some years, significant differences were seen between groups in the number of patients classified as nonadherent or partially adherent; however, the magnitude of difference was less than 5 percentage points. Each year, the mean [SD] number of pharmacy visits to fill ART prescriptions was significantly greater for pilot pharmacy patients compared with nonpilot pharmacy patients (e.g., 2007: 15.2 [9.8] vs. 13.7 [6.8] respectively, $P<0.001$ ); however, the median number of visits (13) was the same for both groups each year.

A significantly greater percentage of pilot pharmacy patients used a PI-based ART regimen (NRTI $+\mathrm{PI} \pm \mathrm{NNRTI}$ ) compared with nonpilot patients during 2005 and 2007 (Table 3). A significantly smaller percentage used an NNRTI-based regimen (NRTI + NNRTI) compared with nonpilot patients during 2007 (21.0\% vs. $26.2 \%$, respectively, $P=0.011$ ), but not during 2005 or 2006. During 2006, no pilot pharmacy patients were taking the contraindicated single NRTI treatment strategy, compared with $10(0.6 \%)$ of nonpilot pharmacy patients $(P=0.050)$; differences in the other years were not significant. Each year, a smaller proportion of pilot pharmacy patients was on contraindicated ART regimens compared with nonpilot pharmacy patients (e.g., 2007: $8.9 \%$ vs. $12.2 \%$ respectively, $P=0.027$ ).

The percentage of patients who used only a single ART medication strategy in a year was significantly greater for pilot pharmacies versus nonpilot pharmacies, with the difference being approximately 22 percentage points each year (e.g., 2007: $71.7 \%$ vs. $49.1 \%$, respectively, $P<0.001$; Table 3). The percentages of patients experiencing opportunistic infections were similar, approximately 35\%, for pilot and nonpilot pharmacy patients each year $(P=0.809-0.945)$. Logistic regression indicated that the most important factors associated with likelihood of adherence were use of a pilot pharmacy 


\section{Antiretroviral Therapy Adherence, Medication Use, and Health Care Costs During 3 Years of a Community Pharmacy Medication Therapy Management Program for Medi-Cal Beneficiaries with HIV/AIDS}

TABLE 3 Prescription Medication Use: Pilot and Nonpilot Pharmacies, 2005-2007

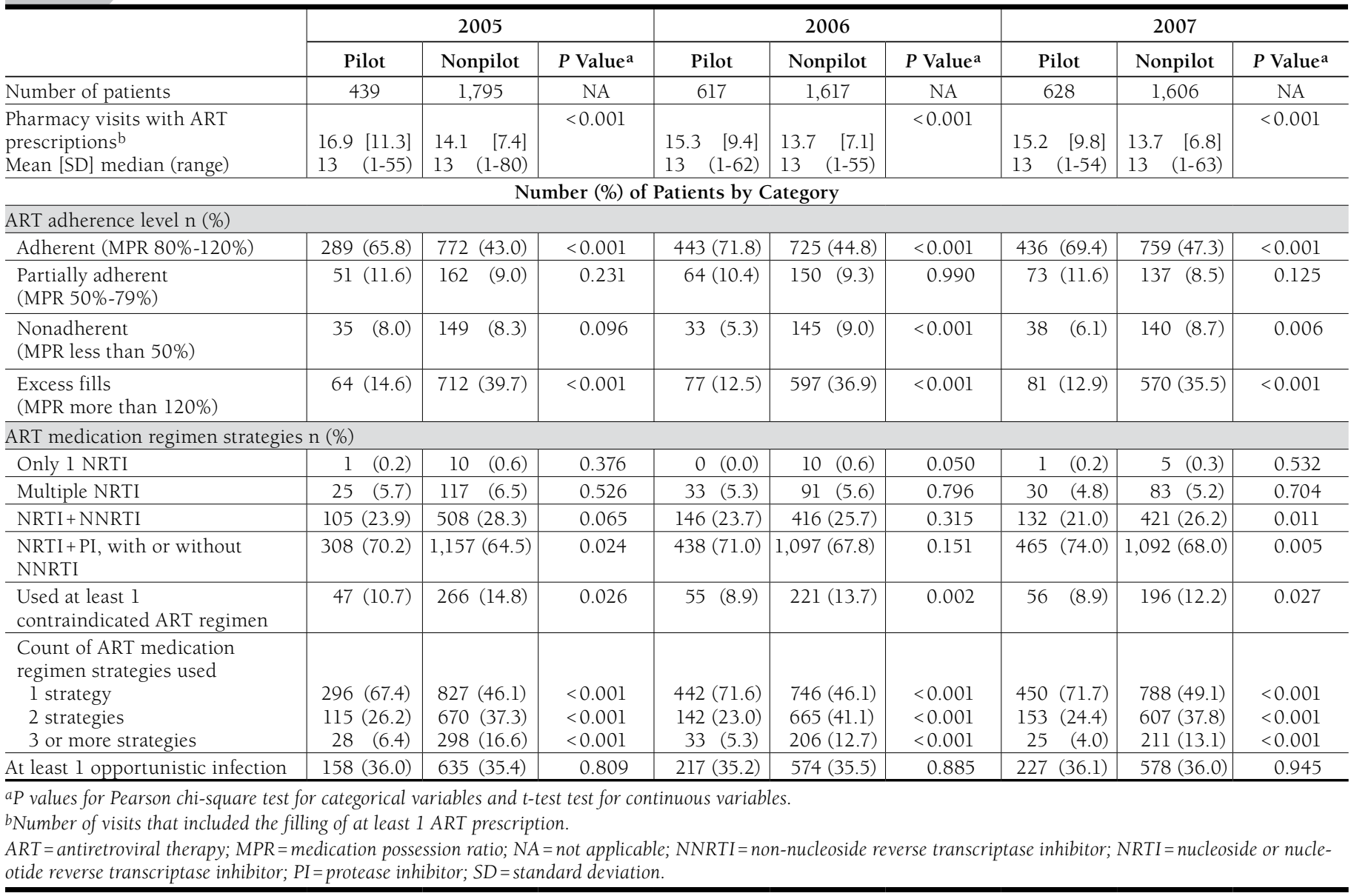

(odds ratio $[\mathrm{OR}]=2.74,95 \% \mathrm{CI}=2.44-3.10$ ), and Latino or African American ethnicity $(\mathrm{OR}=1.45,95 \% \mathrm{CI}=1.25-1.67$ and $\mathrm{OR}=1.15,95 \% \mathrm{CI}=1.03-1.30$ respectively; Table 4).

In the generalized linear model analyses, the predicted mean (standard error [SE]) total costs per patient were not significantly different in the pilot versus other pharmacy group in any year (Table 5). The largest expenditure categories were medications, other services, and inpatient services. During each year, the predicted mean [SE] total medication cost per patient was greater in the pilot group than the nonpilot group (e.g., 2007: \$29,955 [\$679] vs. \$25,690 [\$362], respectively, $P<0.001$ ). The difference was driven largely by the difference in non-ART medication costs, which were approximately $30 \%-40 \%$ greater in pilot pharmacy groups each year (e.g., 2007: \$10,815 [\$538] vs. \$8,190 [\$252] respectively, $P<0.001)$. Examination of the types of non-ART medications utilized (categories with costs exceeding $\$ 500$ per patient in each year, 2005-2007, in either the pilot or nonpilot pharmacy group) indicated that the difference in non-ART costs was primarily and consistently attributable to greater use of

\section{TABLE 4 Logistic Regression Analysis of Factors Associated with ART Adherence, 2005-2007}

\begin{tabular}{|c|c|c|c|c|}
\hline & \multirow{2}{*}{$\begin{array}{l}\text { Odds } \\
\text { Ratio }\end{array}$} & \multicolumn{2}{|c|}{ 95\% Confidence Interval } & \multirow[b]{2}{*}{$P$ Value } \\
\hline & & Lower & Upper & \\
\hline Pilot pharmacy use & 2.74 & 2.44 & 3.10 & $<0.001$ \\
\hline Age (years) & 1.00 & 0.99 & 1.01 & 0.858 \\
\hline Male & 1.06 & 0.95 & 1.17 & 0.300 \\
\hline
\end{tabular}

Ethnicityb

\begin{tabular}{l|l|l|l|r}
\hline African American & 1.15 & 1.03 & 1.30 & 0.017 \\
\hline Latino & 1.45 & 1.25 & 1.67 & $<0.001$ \\
\hline Other & 1.18 & 0.99 & 1.40 & 0.067 \\
\hline
\end{tabular}

aUnit of analysis was the patient-year (i.e., each patient contributed 1 observation for each of the 3 years), with $n=3,424$ in the adherent category (MPR 80\%-120\%) and $n=3,278$ in the other adherence category, which included patients who were nonadherent (MPR less than 50\%), partially adherent (MPR 50\%-79\%), or had excess fills (MPR more than 120\%). R-square $=0.0648 ; c$-statistic $=0.613$.

${ }^{b}$ Ethnicity reference category was Non-Latino White.

$A R T=$ antiretroviral therapy; $M P R=$ medication possession ratio 


\section{Antiretroviral Therapy Adherence, Medication Use, and Health Care Costs During 3 Years of a Community Pharmacy Medication Therapy Management Program for Medi-Cal Beneficiaries with HIV/AIDS}

\section{TABLE 5 Generalized Linear Model Analyses of Predicted Pharmacy and Medical}

Costs Per Patient Controlling for Age, Gender, and Ethnicity, 2005-2007 a

\begin{tabular}{|c|c|c|c|c|c|c|c|c|c|}
\hline \multirow[b]{3}{*}{ Cost category } & \multicolumn{3}{|c|}{2005} & \multicolumn{3}{|c|}{2006} & \multicolumn{3}{|c|}{2007} \\
\hline & \multirow{2}{*}{$\begin{array}{c}\text { Pilot } \\
\text { Mean (SE) } \\
\text { Cost }(\$)\end{array}$} & \multirow{2}{*}{$\begin{array}{c}\text { Nonpilot } \\
\text { Mean (SE) } \\
\text { Cost (\$) }\end{array}$} & \multirow[b]{2}{*}{$P$ Value $^{\mathrm{b}}$} & Pilot & Nonpilot & \multirow[b]{2}{*}{$P$ Value $^{b}$} & \multirow{2}{*}{$\begin{array}{c}\text { Pilot } \\
\text { Mean (SE) } \\
\text { Cost (\$) }\end{array}$} & \multirow{2}{*}{$\begin{array}{c}\text { Nonpilot } \\
\text { Mean (SE) } \\
\text { Cost (\$) }\end{array}$} & \multirow[b]{2}{*}{$P$ Value $^{\mathrm{b}}$} \\
\hline & & & & $\begin{array}{c}\text { Mean }(\mathrm{SE}) \\
\text { Cost }(\$)\end{array}$ & $\begin{array}{l}\text { Mean (SE) } \\
\text { Cost }(\$)\end{array}$ & & & & \\
\hline Total medication & $26,797 \quad(703)$ & $22,544 \quad(290)$ & $<0.001$ & $27,671 \quad(613)$ & $23,190 \quad(315)$ & $<0.001$ & $29,955 \quad(679)$ & $25,690 \quad(362)$ & $<0.001$ \\
\hline ART medication & $16,807 \quad(403)$ & $15,526 \quad(183)$ & 0.003 & $18,022(349)$ & 15,913 (189) & $<0.001$ & $19,041 \quad(375)$ & $17,452 \quad(214)$ & $<0.001$ \\
\hline Non-ART medication & $9,887 \quad(579)$ & $7,014 \quad(200)$ & $<0.001$ & $9,611 \quad(476)$ & 7,253 (219) & $<0.001$ & $10,815 \quad(538)$ & $8,190 \quad(252)$ & $<0.001$ \\
\hline Inpatient & $2,734 \quad(313)$ & $3,808 \quad(207)$ & 0.006 & 3,219 (311) & $4,140(240)$ & 0.021 & $3,083 \quad(293)$ & $5,186 \quad(300)$ & $<0.001$ \\
\hline $\begin{array}{l}\text { Hospital outpatient } \\
\text { including ER }\end{array}$ & $183 \quad(21)$ & $250 \quad(14)$ & 0.011 & $134 \quad(13)$ & $264 \quad(15)$ & $<0.001$ & $96 \quad(9)$ & $248 \quad(14)$ & $<0.001$ \\
\hline Outpatient & $112 \quad(11)$ & $44 \quad(2)$ & $<0.001$ & $82 \quad(7)$ & $43 \quad(2)$ & $<0.001$ & (7) & (2) & $<0.001$ \\
\hline Mental health & $366 \quad(49)$ & $182 \quad(11)$ & $<0.001$ & $292 \quad(37)$ & $309 \quad(22)$ & 0.698 & 259 & $(27)$ & 0.008 \\
\hline Lab and x-ray & $389 \quad(34)$ & (17) & 0.736 & $387 \quad(28)$ & (18) & 0.530 & (29) & (18) & 0.974 \\
\hline $\begin{array}{l}\text { AIDS-alternative to nurs- } \\
\text { ing facility or hospital }\end{array}$ & 912 (123) & $894 \quad(58)$ & 0.883 & 859 (98) & $819 \quad(56)$ & 0.709 & $815 \quad$ (91) & (58) & 0.813 \\
\hline Other & $3,626 \quad(274)$ & $5,044 \quad(185)$ & $<0.001$ & $3,903(250)$ & $5,572 \quad(217)$ & $<0.001$ & $4,050 \quad(254)$ & $5,322 \quad(206)$ & $<0.001$ \\
\hline Total & $35,546(1,093)$ & $33,501 \quad(505)$ & 0.079 & $36,806(980)$ & $35,230(575)$ & 0.157 & $38,983(1,023)$ & $38,856 \quad(633)$ & 0.915 \\
\hline
\end{tabular}

gastrointestinal agents, analgesics, and psychotherapeutic drugs in the pilot pharmacy group compared with the nonpilot pharmacy group (Table 6).

The 2 remaining large expenditure categories, inpatient and other services, were significantly lower for pilot patients than nonpilot patients each year (Table 5). For example, predicted mean (SE) expenditures for inpatient services in 2007 for the pilot and nonpilot groups were $\$ 3,083$ (\$293) versus $\$ 5,186$ $(\$ 300)$, respectively $(P<0.001)$. Although significantly lower costs for other services were observed for the pilot pharmacy group compared with the other pharmacy group each year $(P<0.001)$, the difference was not consistently attributable to a few specific services; the other category included more than 30 types of services, such as professional services provided by allied health care providers (e.g., chiropractor, optometrist, and psychologist), and facility services (e.g., adult day care, rehabilitation centers, and skilled nursing facilities). Although not a large contributor to total cost, the mean (SE) costs per patient for hospital outpatient services (which includes emergency room visits) were significantly lower for pilot than nonpilot pharmacy patients during each year (e.g., 2007: \$96 [\$9] vs. $\$ 248$ [\$14] respectively, $P<0.001$ ).

During the 3-year study period, payments by the California DHCS to pilot pharmacies for MTM services provided to study patients were $\$ 430,141$ in 2005 ( $n=439$ patients); $\$ 620,284$ in $2006(n=617)$; and $\$ 657,752$ in $2007(n=628)$. Thus, the average annual DHCS payment for MTM services per pilot pharmacy study patient was about $\$ 1,000$ each year $(\$ 980, \$ 1,005$, and $\$ 1,047$, respectively).

\section{Discussion}

The present study assessed the results of a novel compensation program for community pharmacies offering MTM services for patients with HIV/AIDS enrolled in Medi-Cal over a 3-year period. Overall, the results of our initial evaluation (2005 data only, and including 53.3\% Medicare dual-eligibles), ${ }^{10}$ were sustained in this group of patients who continued to receive care at pilot pharmacies through Medi-Cal from January 1, 2005, through December 31, 2007.

A greater percentage of pilot pharmacy patients were adherent to their ART medication regimens compared with patients using nonpilot pharmacies, with the difference being greater than 20 percentage points each year. After controlling for age, gender, and ethnicity, the odds of adherence for pilot pharmacy patients were more than twice those of nonpilot pharmacy patients. The fact that the proportion of patients who were partially adherent or nonadherent remained fairly constant in both groups is troublesome because these patients are at risk of developing ART resistance that could diminish their longerterm therapy options. As in 2005, a much smaller percentage of patients using pilot pharmacies had excess fills than patients using other pharmacies during 2006 and 2007. A reasonable expectation is that the greater percentage of adherent patients in pilot pharmacies will result in improved clinical outcomes given studies that have reported an association of improved adherence with reduced viral load. ${ }^{5-8}$

There were some slight differences in the percentage of males and the race/ethnicity mix of pilot versus nonpilot pharmacies in 2006 and 2007, with pilot pharmacies having fewer males, fewer non-Latino White patients, and more Latino 


\section{Antiretroviral Therapy Adherence, Medication Use, and Health Care Costs During 3 Years of a Community Pharmacy Medication Therapy Management Program for Medi-Cal Beneficiaries with HIV/AIDS}

TABLE 6 Generalized Linear Model Analyses of Predicted Selected Non-ART Medication Costs Per Patient by Therapeutic Category Controlling for Age, Gender, and Ethnicity, 2005-2007ª

\begin{tabular}{|c|c|c|c|c|c|c|c|c|c|}
\hline \multirow{3}{*}{ Medication category } & \multicolumn{3}{|c|}{2005} & \multicolumn{3}{|c|}{2006} & \multicolumn{3}{|c|}{2007} \\
\hline & \multirow{2}{*}{$\begin{array}{l}\text { Pilot } \\
\text { Mean (SE) } \\
\text { Cost }(\$)\end{array}$} & \multirow{2}{*}{$\begin{array}{l}\text { Nonpilot } \\
\text { Mean (SE) } \\
\text { Cost }(\$)\end{array}$} & \multirow[b]{2}{*}{$P$ Value ${ }^{b}$} & Pilot & Nonpilot & \multirow[b]{2}{*}{$P$ Value ${ }^{b}$} & Pilot & Nonpilot & \multirow[b]{2}{*}{$P$ Value $^{\mathrm{b}}$} \\
\hline & & & & $\begin{array}{l}\text { Mean (SE) } \\
\text { Cost (\$) }\end{array}$ & $\begin{array}{l}\text { Mean }(\mathrm{SE}) \\
\text { Cost }(\$)\end{array}$ & & $\begin{array}{l}\text { Mean (SE) } \\
\text { Cost (\$) }\end{array}$ & $\begin{array}{l}\text { Mean (SE) } \\
\text { Cost }(\$)\end{array}$ & \\
\hline $\begin{array}{l}\text { Gastrointestinal (e.g., antiemetic/antiver- } \\
\text { tigo, gastric acid secretion reducers) }\end{array}$ & $1,793(203)$ & $1,016 \quad(55)$ & $<0.001$ & $1,836(175)$ & $1,173 \quad(68)$ & $<0.001$ & $1,972(186)$ & $1,385(80)$ & $<0.001$ \\
\hline Analgesics & $1,725(186)$ & $505(26)$ & $<0.001$ & $1,330(119)$ & $536 \quad(29)$ & $<0.001$ & $1,563(142)$ & $766 \quad(42)$ & $<0.001$ \\
\hline $\begin{array}{l}\text { Psychotherapeutic drugs (e.g., atypical } \\
\text { antipsychotics, antidepressants, anti- } \\
\text { anxiety agents) }\end{array}$ & $1,297(137)$ & $907 \quad(46)$ & 0.001 & $1,262(113)$ & 951 (51) & 0.005 & $1,372(123)$ & $1,069 \quad(58)$ & 0.014 \\
\hline Anti-infectives/miscellaneous & $1,016(111)$ & $1,089 \quad(57)$ & 0.549 & $991 \quad(94)$ & $767(44)$ & 0.018 & $949(87)$ & $784 \quad(44)$ & 0.069 \\
\hline CNS (primarily anticonvulsants) & $528 \quad(62)$ & $434 \quad(24)$ & 0.113 & $556 \quad(55)$ & $436 \quad(26)$ & 0.028 & $596 \quad(58)$ & $482 \quad(29)$ & 0.055 \\
\hline \multicolumn{10}{|c|}{$\begin{array}{l}\text { aCategories exceeding } \$ 500 \text { per patient in } 2005-2007 \text { in either pilot or other pharmacy group. } N \text { of cases }=2,208 \text { in each year. Goodness-of-fit statistics for } 2007 \text { : Pearson } \\
\text { chi-square, gastrointestinal }=7.6584 \text {, analgesics }=15.0464, \text { psychotherapeutic }=4.4078 \text {, anti-infectives }=12.7998, C N S=5.4685 \text {. Results were similar for } 2005 \text { and } 2006 \text {. } \\
\text { bAssessed using Wald chi-square test for regression coefficient. } \\
\text { ART=antiretroviral therapy; CNS = central nervous system; SE = standard error. }\end{array}$} \\
\hline
\end{tabular}

patients than nonpilot pharmacies, although the largest difference was less than 10 percentage points. Therefore, in this study cohort, the demographic profile of HIV/AIDS patients receiving medications at pilot pharmacies was generally similar to that of HIV/AIDS patients receiving medications at nonpilot pharmacies in California.

In 2005 and 2007, a larger percentage of pilot pharmacy compared with nonpilot pharmacy patients used PI-based ART medication regimens, although in 2006 the difference was not statistically significant. Each year, the percentages of patients using only a single ART medication strategy were greater for pilot pharmacy patients, and fewer pilot pharmacy patients were on contraindicated regimens. Remaining on a single type of medication regimen strategy is important because exposing patients to more drugs and drug classes increases the likelihood of developing ART resistance. These findings suggest that ART medications were being managed more successfully in pilot pharmacy patients, giving them more future treatment options.

Contrary to our hypotheses, 2 outcomes (opportunistic infection rate and total annual cost per patient) were not significantly lower in pilot pharmacy patients. The proportion of patients experiencing opportunistic infections (approximately one-third) was similar between the 2 groups each year despite having a greater proportion of adherent patients and patients on PI-based regimens in pilot versus nonpilot pharmacies. Although the reasons for this finding cannot be determined from our data, it could be lack of sufficient follow-up time to develop opportunistic infections which are usually observed in patients with advanced disease. Although the total annual cost per patient was similar in each group each year (e.g., approximately $\$ 39,000$ in 2007), the mix of expenditures differed. Non-ART medication costs were appreciably greater in the pilot pharmacy group each year, while expenditures for inpatient services were significantly lower for pilot than nonpilot patients. Higher non-ART medication use comprised greater use of gastrointestinal agents (e.g., antiemetics and antivertigo agents), analgesics, and psychotherapeutic medications. The greatest magnitude of difference was observed for analgesics, for which the average expenditure per pilot pharmacy patient was more than twice that of a nonpilot pharmacy patient during each of the 3 years. Analgesics are often used to treat pain of peripheral neuropathy, a common neurological complication associated with HIV infection. Antiemetics are often used to treat nausea, a side effect that may occur with ART medications. Thus, pilot pharmacy patients appear to be receiving more non-ART medications to mitigate commonly occurring adverse effects of HIV and its treatment. This finding is consistent with the reduction in drug-related toxicities reported for HIV patients receiving MTM services in a primary care clinic. ${ }^{9}$ Thus, the role of the pharmacist in proactively managing adverse drug reactions may increase medication costs but improve compliance and long-term patient outcomes.

Including the additional amount paid by the California DHCS (average of approximately $\$ 1,000$ per pilot pharmacy study patient per year because of the $\$ 9.50$ per prescription payments for MTM services) would increase the total cost for pilot patients by less than 3\%. Of note, the California Legislature made changes to the pilot program effective July 1,2008 , requiring that California's DHCS pay the $\$ 9.50$ only for ART prescriptions, not for all prescriptions dispensed, as was done from 2005 through 2007. Under the first year of the new payment scheme (July 1, 2008, through June 30, 2009), the total amount that DHCS paid to pilot pharmacies (for all patients, not just patients in this study) was approximately $40 \%$ of that paid in the previous fiscal year $(\$ 1,012,852$ vs. $\$ 2,607,313$, respectively). Applying this reduction to the average additional amount per patient paid per study patient in 


\section{Antiretroviral Therapy Adherence, Medication Use, and Health Care Costs During 3 Years of a Community Pharmacy Medication Therapy Management Program for Medi-Cal Beneficiaries with HIV/AIDS}

2007 for MTM services (based on all prescriptions) would result in an estimated average payment of $\$ 400$ per patient, which would add approximately $1 \%$ to the total cost of pilot pharmacy patients. Examination of data in subsequent years would be needed to determine if total costs remain similar in each group. Additionally, if our finding that more pilot patients are being maintained successfully on a single (or fewer) treatment regimen each year extends over many years, then the ART drug costs in the pilot group longitudinally may remain relatively stable over time, while the nonpilot pharmacy group ART medication costs would be expected to increase as the nonpilot pharmacy patients utilize more ARTs, newer ARTs, and more intensive salvage regimens.

Although this study spanned a 3-year period, the ultimate long-term benefit of patients being more adherent on appropriate combined ART regimens and utilizing more non-ART medications would require longer follow-up and remains to be studied. ${ }^{12}$ Regardless of whether cost offsets occur, the full value realized from the resources devoted to pilot pharmacy patients should ultimately be assessed on the basis of qualityadjusted life years (QALYs) gained over time. Since HIV is now considered a chronic disease that is treatable over decades, outcomes beyond medical claims data, such as maintenance of employment, fewer sick days, improved productivity, and patients' quality of life should be explicitly considered and measured.

\section{Limitations}

First, the present study was an evaluation of an ongoing program and as such may be subject to selection bias, since patients were not randomly assigned to pilot versus nonpilot pharmacies. Although the 2 groups of patients were similar with regard to demographics, it is not possible to clearly determine to what extent the results we have observed are due to the true pilot program effect or to unobserved differences in patients using pilot versus nonpilot pharmacies. Second, a more rigorous quasi-experimental (pre-intervention vs. post-intervention with comparison group) study design was not possible, since pilot pharmacies had been offering MTM services prior to the Medi-Cal compensation program. A third limitation is the availability of only self-reported data regarding MTM services at pilot pharmacies. Data regarding pharmacy services provided at the nonpilot pharmacies, which could have been providing some MTM services beyond the California requirement to offer counseling on new prescriptions, were also unavailable. Although possible, most likely the nonpilot pharmacies did not offer the types of specialized MTM services for patients with HIV/AIDS that would have been comparable to the MTM services provided by the pilot pharmacies. ${ }^{11}$ Fourth, some patients classified as nonpilot pharmacy patients also had prescriptions filled at pilot pharmacies. However, we found that $88.9 \%$ of nonpilot pharmacy patients filled no ART prescriptions at pilot pharmacies, and despite this crossover usage, we still noted differences in the nonpilot versus pilot pharmacy patients.

Fifth, as in any study that relies on retrospective analysis of administrative claims data, use of the Medi-Cal database does not allow measurement of important clinical outcomes, such as viral load and immune status, which would be indicative of patients' clinical status and disease progression or severity. Sixth, patients who had been dually eligible for Medicare were automatically switched to Medicare Part D for their prescription drugs as of January 1, 2006, and thus were not available for follow-up in this Medi-Cal database, although they may continue to be patients of the pilot pharmacies. Similarly, the Medi-Cal patients do not represent the entire patient load of either the pilot or nonpilot pharmacies. Thus, results may not generalize to patient populations covered by Medicare or by commercial insurance, which might differ from the present study sample with respect to demographics, disease characteristics, and medical care patterns.

\section{Conclusions}

Over a 3-year intervention period, patients with HIV/AIDS filling ART prescriptions at MTM pilot pharmacies consistently had higher ART adherence rates, fewer excess fills, and fewer contraindicated regimens than nonpilot pharmacy patients. In addition, more pilot pharmacy patients remained on a single type of ART regimen each year of the study, thus decreasing the likelihood of developing drug resistance and preserving therapeutic options in the future. There were no significant differences between the 2 groups in mean total all-cause health care cost per patient. The results of this novel pilot program should help health care plans understand the possible value of MTM for patients with HIV/AIDS and spur continued evaluation of MTM services provided in community pharmacies.

\section{Authors}

JAN D. HIRSCH, RPh, PhD, is Associate Professor of Clinical Pharmacy, Skaggs School of Pharmacy and Pharmaceutical Sciences; and BROOKIE M. BEST, PharmD, MAS, is Associate Professor of Clinical Pharmacy and Pediatrics, Skaggs School of Pharmacy and Pharmaceutical Sciences and School of Medicine, University of California, San Diego, La Jolla, California. MARCO GONZALES, PharmD, and TERESA ANN MILLER, PharmD, are Senior Pharmaceutical Consultants, California Department of Health Care Services, Sacramento, California. ASHLEY ROSENQUIST, PharmD, is Clinical Pharmacist, Intermountain Medical Center, Murray, Utah. TODD P. GILMER, PhD, is Professor, Department of Family and Preventive Medicine, University of California, San Diego, La Jolla, California.

AUTHOR CORRESPONDENCE: Jan D. Hirsch, RPh, PhD, Skaggs School of Pharmacy and Pharmaceutical Sciences, University of California, San Diego, 9500 Gilman Dr., Mail Code 0714, La Jolla, CA 92093-0714. Tel: 858.822.5562; Email: janhirsch@ucsd.edu. 


\section{Antiretroviral Therapy Adherence, Medication Use, and Health Care Costs During 3 Years of a Community Pharmacy Medication Therapy Management Program for Medi-Cal Beneficiaries with HIV/AIDS}

\section{DISCLOSURES}

Payment to pharmacies participating in the pilot program for MTM services was funded by the State of California. There was no other external funding for this research. Two authors are employed by the California Department of Health Care Services, Medi-Cal Pharmacy Policy Branch. This department had responsibility for implementing the pilot MTM services program that is evaluated in this research.

Concept and design were performed by Gilmer, Hirsch, Miller, and Rosenquist. Data were collected by Gonzales, with the assistance of Miller and Hirsch, and interpreted by Best, with the assistance of Hirsch and Gilmer. The manuscript was written by Hirsch, with the assistance of Gonzales, Gilmer, and Rosenquist, and revised by Best, with the assistance of Gonzales and Hirsch.

\section{REFERENCES}

1. U.S. DHHS Panel on Antiretroviral Guidelines for Adults and Adolescents. Guidelines for the use of antiretroviral agents in HIV-1-infected adults and adolescents. U.S. Department of Health and Human Services. January 10, 2011. Available at: http://www.aidsinfo.nih.gov/ContentFiles/ AdultandAdolescentGL.pdf. Accessed March 14, 2011.

2. Paterson DL, Swindells S, Mohr J, et al. Adherence to protease inhibitor therapy and outcomes in patients with HIV infection. Ann Intern Med. 2000;133(1):21-30. Available at: http://www.annals.org/ content/133/1/21.1.full.pdf. Accessed March 14, 2011.

3. Sethi AK, Celentano DD, Gange SJ, Moore RD, Gallant JE. Association between adherence to antiretroviral therapy and human immunodeficiency virus drug resistance. Clin Infect Dis. 2003;37(8):1112-18.

4. Wood E, Hogg RS, Yip B, Harrigan PR, O'Shaughnessy MV, Montaner JS. Is there a baseline CD4 cell count that precludes a survival response to modern antiretroviral therapy? AIDS. 2003;17(5):711-20.

5. Inciardi JF, Leeds AL. Assessing the utility of a community pharmacy refill record as a measure of adherence and viral load response in patients infected with human immunodeficiency virus. Pharmacotherapy. 2005;25(6):790-96

6. Fairley CK, Permana A, Read TR. Long-term utility of measuring adherence by self-report compared with pharmacy record in a routine clinic setting. HIV Med. 2005;6(5):366-69.

7. Grossberg R, Zhang Y, Gross R. A time-to-prescription-refill measure of antiretroviral adherence predicted changes in viral load in HIV. J Clin Epidemiol. 2004;57(10):1107-10

8. Cantwell-McNelis K, James CW. Role of clinical pharmacists in outpatient HIV clinics. Am J Health Syst Pharm. 2002;59(5):447-52.

9. March K, Mak M, Louie SG. Effects of pharmacists' interventions on patient outcomes in an HIV primary care clinic. Am J Health Syst Pharm. 2007;64(24):2574-78.

10. Hirsch JD, Rosenquist A, Best BM, Miller TA, Gilmer TP. Evaluation of the first year of a pilot program in community pharmacy: HIV/AIDS medication therapy management for Medi-Cal beneficiaries. J Manag Care Pharm. 2009;15(1):32-41. Available at: http://www.amcp.org/data/jmcp/032-04l.pdf.

11. Rosenquist A, Best BM, Miller TA, Gilmer TP, Hirsch JD. Medication therapy management services in community pharmacy: a pilot programme in HIV specialty pharmacies. J Eval Clin Pract. 2010;16(6):1142-46.

12. Palella FJ Jr, Delaney KM, Moorman AC, et al. Declining morbidity and mortality among patients with advanced human immunodeficiency virus infection. HIV Outpatient Study Investigators. N Engl J Med. 1998;338(13):853-60. Available at: http://www.nejm.org/doi/pdf/10.1056/ NEJM199803263381301. Accessed March 14, 2011.

\section{APPENDIX}

Antiretroviral Therapy

Medication List by Category

Nucleoside and Nucleotide Reverse Transcriptase Inhibitors (NRTI)

Abacavir sulfate

Abacavir sulfate/lamivudine

Abacavir/lamivudine/zidovudine

Didanosine/mag/Al NaCB/sodium cit ${ }^{\mathrm{a}}$

Didanosine

Didanosine/calcium carbonate/magnesium

Didanosine/sodium citrate

Emtricitabine

Lamivudine

Lamivudine/zidovudine

Stavudine

Zalcitabine

Zidovudine

Tenofovir disoproxil fumarate

Emtricitabine/tenofovir

Non-Nucleoside Reverse Transcriptase Inhibitors (NNRTI)

Delavirdine mesylate

Efavirenz

Nevirapine

\begin{tabular}{l}
\hline \multicolumn{1}{c}{ Protease Inhibitors (PI) } \\
\hline Amprenavir/vitamin E \\
\hline Amprenavir/vitamin E/propylene glycol \\
\hline Atazanavir sulfate \\
\hline Fosamprenavir calcium \\
\hline Indinavir sulfate \\
\hline Nelfinavir mesylate \\
\hline Ritonavir \\
\hline Ritonavir/lopinavir \\
\hline Saquinavir \\
\hline Saquinavir mesylate \\
\hline Tipranavir \\
\hline Fusion Inhibitor \\
\hline $\begin{array}{l}\text { anfuvfering agents, magnesium hydroxide, aluminum sodium carbonate, sodium } \\
\text { citrate. }\end{array}$ \\
\hline
\end{tabular}

